\title{
The Mitogen-Activated Protein Kinase Signal Transduction Pathways in Alternaria Species
}

\author{
Houjuan $\mathrm{Xu}^{1}$, Xiaoxue $\mathrm{Xu}^{1}$, Yujun Wang ${ }^{1}$, Vivek K. Bajpai ${ }^{2}$, Lisha Huang ${ }^{1}$, Yongfang $\mathrm{Chen}^{1}$ and \\ Kwang-Hyun Baek ${ }^{2 *}$ \\ ${ }^{I}$ College of Plant Protection, Shandong Agricultural University, Tai-an 271-018, China \\ ${ }^{2}$ School of Biotechnology, Yeungnam University, Gyeongsan 712-749, Korea
}

(Received on April 3, 2012; Revised on May 14, 2012; Accepted on May 15, 2012)

\begin{abstract}
Mitogen-activated protein kinase (MAPK) cascades are conserved signaling modules in the eukaryotic cells. They are involved in many major cell processes in fungi such as stress responses, vegetative growth, pathogenicity, secondary metabolism and cell wall integrity. In this review, we summarized the advances of research on the MAPK signaling pathways in Alternaria species. As major phytopathogenic fungi, Alternaria species reduce crop production. In contrast to the five MAPK pathways known in yeast, only three MAPK pathways as Fus3/Kss1-type, Hog1-type, and Slt2-type have been characterized in Alternaria. The Fus3/Kss 1-type MAPK pathway participates in regulation of vegetative growth, conidiation, production of some cell-wall-degrading enzymes and pathogenicity. The Hogl-type pathway is involved in osmotic and oxidative stress, fungicides susceptibility and pathogenicity. The Slt2-type MAP kinases play an important role on maintaining cell wall integrity, pathogenicity and conidiation. Although recent advances on the MAPK pathways in Alternaria spp. reveal many important features on the pathogenicity, there are many unsolved problems regarding to the unknown MAP kinase cascade components and network among other major signal transduction. Considering the economic loss induced by Alternaria spp., more researches on the MAPK pathways will need to control the Alternaria diseases.
\end{abstract}

Keywords : Alternaria species, mitogen-activated protein kinase (MAPK), pathogenicity, signal transduction

Fungi live in a continuous changing environment and receive a lot of external stimuli. In order to survive in this environment and colonize in their hosts, they have developed highly complicated survival strategies. By sensing stimulisignals from outside and translating them into changes in

\footnotetext{
*Corresponding author.

Phone)+82-53-810-3029, FAX) +82-53-810-4769

E-mail)khbaek@ynu.ac.kr
}

gene expression, fungi thus respond to the environmental stimuli (Kiel et al., 2010). There are many signal transduction cascades in fungi, including mitogen-activated protein (MAP) kinase signaling cascades (MAPK pathways), Gprotein regulated cyclic-AMP signaling pathways (PKA pathway), calcium-mediated signaling, Ras1 signaling and so on (Lengeler et al., 2000). These signal transduction pathways control a wide variety of processes in fungi, and furthermore, allow fungi cells to quickly adapt to a changing environment for the survival. Among them, the MAP kinase pathways are widely studied in different fungi and demonstrated their important roles on processes such as responding to biotic and abiotic stresses, mating and pathogenicity (Tables 1, 2 and 3). These diverse functions especially in fungal pathogenicity makes them potentially targets of future antifungal drugs, therefore receive more and more attentions (May et al., 2005).

The MAP kinases (EC 2.7.11.24) are a family of serine/ threonine-specific protein kinases, which are evolutionarily conserved in eukaryotic organisms. These kinases are activated through the protein kinase cascades and respond to diverse external stimuli. For each cascade, there are at least three essential enzymes, a MAP kinase kinase kinase (MAPKKK, MKKK, MEKK or MAP3K), a MAP kinase kinase (MAPKK, MKK, MEK, or MAP2K) and a MAP kinase (MAPK) which are activated in series. By sequential phosphorylation, MAPKKKs receive environmental signals and deliver them to MAPKKs, then to MAPKs. Finally the phosphorylated MAPKs deliver these signals to downstream targets, including several kinases and transcription factors, which eventually regulate some genes gene expression responding to the external stimulus (Chen and Thorner, 2007).

Over the past decades extensive numbers of research have revealed remarkable functions of MAPK pathway in fungi, especially in Saccharomyces cerevisiae which have five MAPK pathways for pheromone response, filamentation/invasion, high osmolarity/growth, cell integrity, and spore cell assembly (Gustin et al., 1998). For phytopathogenic fungi, the most extensively studied organism is the 
rice blast fungus Magnaporthe grisea. Three MAPK signaling pathways named as pmk1, mps 1 and osm 1 play diverse roles in pathogenesis-related development (Dixon et al., 1999; Xu and Hamer, 1996; Xu et al., 1998). Other plant pathogens, e.g., Colletotrichum lagenarium (Kojima et al., 2002; Kojima et al., 2004; Takano et al., 2000), Botrytis cinerea (Liu et al., 2011; Rui and Hahn, 2007; Segmuller et al., 2006) and Mycosphaerella graminicola (Cousin et al., 2006; Mehrabi et al., 2006a; Moriwaki et al., 2006) have also been studied in depth on the functions of their MAPK signaling pathways.

Alternaria is a large genus of moulds that includes saprophytic, endophytic and pathogenic species. It contains about 50 species of which most are plant parasites. These plant pathogens can infect a large number of economically important plants, and significant reduce yields quantitatively and qualitatively (Verma and Saharan, 1994). Alternaria spp. cause at least $20 \%$ of agricultural spoilage and even cause many human health disorders (Sobiya et al., 2012).
Major plant diseases caused by these fungi include early blight of potato by A. alternata, stem canker of tomato by $A$. arborescens, leaf lesions on Asian pear by $A$. arbusti, brassica dark leaf spot on most Brassica species by $A$. brassicicola, brown spot disease on tobacco by $A$. longipes, Alternaria blight of ginseng by A. panax, and early blight in potatoes and tomatoes by $A$. solani.

Previous studies on fungi reveal the important roles of MAPK signaling pathways in fungal development and virulence, but the processes regulated by same type of MAPK pathways in different fungi are as diverse as the fungi themselves (Tables 1, 2, 3). In recent years, a lot of work has been done to understand the roles of this conserved MAPK pathway cascades in Alternaria species. These extensive works have been resulted in isolation and functional characterization of many genes of the three MAPK pathways (Fus3/Kss1-type, Hogl-type, and Slt2type) in Alternaria (Fig. 1), which includes three MAPK proteins of Amk1, AbSlt2 and AbHog1 in A. brassicicola

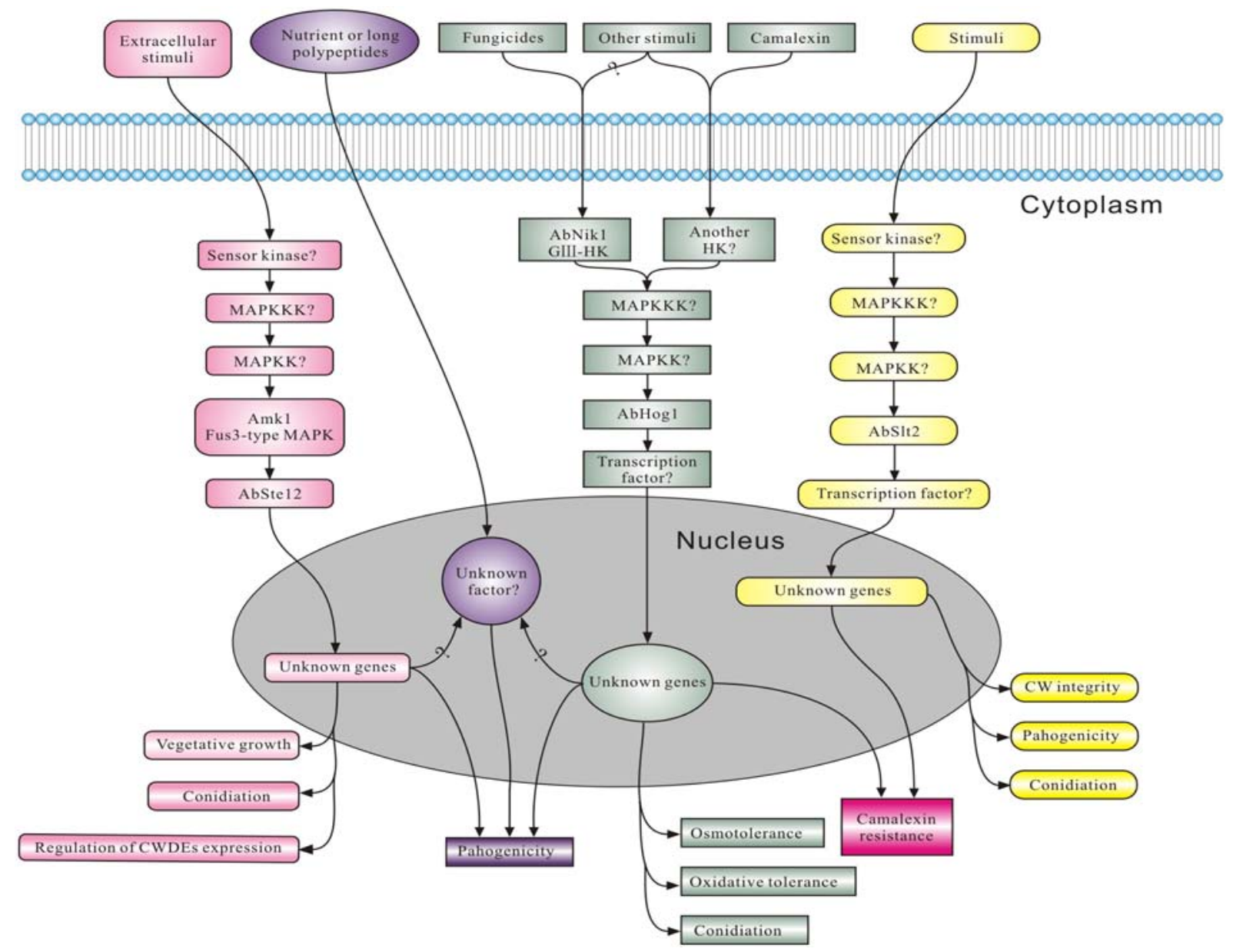

Fig. 1. The mitogen-activated protein kinase (MAPK) pathways in A. brassicicola. Only three MAPK pathways (Fus3/Kss1-type, Hog1type, and Slt2-type) and the corresponding MAPK proteins such as Amk1, AbSlt2 and AbHog1 have been characterized in $A$. brassicicola. The upstream components of MAPKKK and MAPKK and downstream components except AbSte12 in the Fus3/Kss1type pathway have not been characterized in Alternaria. 
and AaFus3, AaSlt2 and AaHog1 in A. alternata. In Fus3/ Kss1-type pathway, the extracellular stimuli pass the signals sequentially to senor kinases, MAPKKK, MAPKK, and MAPK Amk1. The Amk1 further transmits the signals to transcription factor AbSte12, and the AbSte12 turns on genes with the roles on vegetative growth, conidiation, and pathogenicity. In the Hog1-type pathway, AbNik1 detects extracellular fungicides and other stimuli and another histidine kinase may detect camalexin and external stimuli, and the signals are transmitted to AbHog1 through MAPKKK and MAPKK. The AbHog1 is transported to the nucleus and interacts with unknown transcription factors for tolerance induction of osmotic and oxidative stresses. The AbSlt 2 protein transmits the signals transferred from the corresponding unkown MAPKK to nucleus by interacting with unknown transcription factors. The three MAPK signaling pathways work independently, or sometimes act coordinately to form signaling networks.

There is a limited resource delivering the recent advances of the research on MAPK signaling pathways in Alternaria

Table 1. Fus3/Kss 1 homologs and their functions

\begin{tabular}{|c|c|c|c|c|}
\hline Fungal Species & Gene & $\begin{array}{l}\text { Genebank } \\
\text { accession no. }\end{array}$ & Functions & References \\
\hline A. alternata & AaFus3 & ACY73851.1 & $\begin{array}{l}\text { Vegetative growth, conidiation, pathogenicity, expression } \\
\text { of CWDEs }\end{array}$ & (Lin and Chung, 2010) \\
\hline A. brassicicola & Amk1 & - & $\begin{array}{l}\text { Vegetative growth, conidiation, pathogenicity, appreso- } \\
\text { rium production, expression of CWDEs }\end{array}$ & (Cho et al. 2007) \\
\hline Aspergillus nidulaus & MpkB & AAF12815 & $\begin{array}{l}\text { Post-karyogamy process, hyphal anastomosis, natural } \\
\text { product biosynthesis }\end{array}$ & (Jun et al., 2011) \\
\hline B. cinerea & Bmp1 & AAG23132 & Growth rate, pathogenicity, host penetration & (Zheng et al., 2000) \\
\hline B. oryzae & Bmk1 & BAD42855 & Conidiation, pathogenicity & (Moriwaki et al., 2007) \\
\hline Beauveria bassiana & Bbmpk1 & AAQ01000 & $\begin{array}{l}\text { Appressorium formation, host penetration, adhesion to } \\
\text { insect cuticles }\end{array}$ & (Zhang et al., 2010) \\
\hline C. parasitica & Cpmk2 & AAP86959 & Conidiation, vegetative growth, virulence & (Choi et al., 2005) \\
\hline C. purpurea & Cpmk1 & CAC47939 & Pathogenicity, & (Mey et al., 2002b) \\
\hline C.heterostrophus & Chk1 & AAF05913 & $\begin{array}{l}\text { Conidiation, appressorium formation, pathogenicity, regu- } \\
\text { lation of cellulase-encoding genes, melanin biosynthesis }\end{array}$ & $\begin{array}{l}\text {-(Eliahu et al., 2007; } \\
\text { Lev et al., 1999) }\end{array}$ \\
\hline C.lagenarium & Cmk1 & AAD50496 & $\begin{array}{l}\text { Appressorium formation, conidial germination, pathoge- } \\
\text { nicity, melanin metabolism }\end{array}$ & (Takano et al., 2000) \\
\hline Candida albicans & Cek1 & P28869 & Mating, filamentation, construction of the cell wall & $\begin{array}{l}\text { (Csank et al., 1998; } \\
\text { Eisman et al., 2006) }\end{array}$ \\
\hline C. albicans & Cek2 & AAG43110 & Mating & (Chen et al., 2000) \\
\hline F. oxysporum & Fmk1 & AAG01162 & $\begin{array}{l}\text { Pathogenicity, invasive growth, root attachment, expres- } \\
\text { sion of CWDEs }\end{array}$ & (Di Pietro et al., 2001) \\
\hline F. graminearum & Gpmk1 & AAL73403 & Pathogenicity, conidiation, expression of CWDEs & (Jenczmionka et al., 2003) \\
\hline $\begin{array}{l}\text { Gaeumannomyces } \\
\text { graminis }\end{array}$ & Gmk1 & AAG44657 & Complement of $M$. grisea Pmkl mutants & $\begin{array}{l}\text { (Dufresne and Osbourn, } \\
\text { 2001) }\end{array}$ \\
\hline M. grisea & Pmk1 & AAC49521 & Appressorium formation, plant infection, pathogenicity & (Xu and Hamer, 1996) \\
\hline M.graminicola & MgFus3 & AAX81518 & Pycnidiation, pathogenicity, stomatal penetration & (Cousin et al., 2006) \\
\hline N. crassa & Mak-2 & AAK25816 & Hyphal fusion, conidiation, mating and vegetative growth & $(\mathrm{Li}, 2005)$ \\
\hline P. teres & Ptk1 & AAK52840 & Conidiation, appressorium formation, and pathogenicity & (Ruiz-Roldan et al., 2001) \\
\hline Puccinia striiformis & $\begin{array}{l}\text { PsMAPK } \\
1\end{array}$ & HM535614 & Regulation of plant penetration and infectious growth & (Guo et al., 2011) \\
\hline S. cerevisiae & Fus3 & NP_009537 & Mating & (Gustin et al., 1998) \\
\hline Sclerotinia sclerotiorum & Smk1 & AAQ54908 & Sclerotial development & (Chen et al., 2004) \\
\hline Stagonospora nodorum & Mak2 & AAX63387 & Vegetative growth, virulence & (Solomon et al., 2005) \\
\hline T. virens & TmkA & EHK23252 & Biocontrol properties, conidiation, expression of CWDEs & $\begin{array}{l}\text { (Mukherjee et al., 2003; } \\
\text { Mendoza-Mendoza, 2003) }\end{array}$ \\
\hline U. maydis & Kpp2 & AAF15528 & Mating, virulence & (Muller et al., 1999) \\
\hline U. maydis & Kpp6 & CAD43731 & Host penetration, mating, virulence & (Brachmann et al., 2003) \\
\hline Verticillium dahliae & Vmk1 & AAW71477 & Conidiation, microsclerotia formation, athogenicity & (Rauyaree et al., 2005) \\
\hline
\end{tabular}


species, although Alternaria is causing serious problems on crop production. Here, we summarize the recent achievements gained on the Alternaria MAPK signaling pathways, and discuss the significance for the regulation and function of these pathways by comparing the composing components with other phytopathogenic fungal species.

\section{The Fus3/Kss1 MAPK pathway}

The Fus3/Kss 1 pathway is the most extensively studied MAPK cascades in fungal pathogens. A large number of FUS3/KSS1 homologs have been isolated and characterized the functions (Table 1). They are involved in appressorium formation, pathogenicity, conidiation, virulence, vegetative growth, melanin metabolism and some other processes. The Fus3/Kss 1 pathways are somewhat functionally conserved but still have diverse roles in different fungi.

The functions of the published fungal FUS3/KSS1 homologs are summarized in Table 1 . As shown in B. cinerea (Zheng et al., 2000) and Neurospora crassa (Li, 2005), the Fus3/Kss 1 pathway has been shown to be required for vegetative growth in Alternaria. Disruption of AaFus3, a $S$. cerevisiae Fus3 homologous gene in A. alternata, decreased the radial growth by $40 \pm 8 \%$ compared to that of wild type on potato dextrose agar (PDA) media, and the growth rate was restored to the original wild type level by reintroducing the gene AaFus3 into the mutants (Lin and Chung, 2010). A similar result was acquired in a study of $A$. brassicicola (Cho et al., 2007). In that study, deletion of Amk1, the AaFus3 homologous gene, also significantly reduced the growth rate of $A$. brassicicola on different media. The regulation of vegetative growth by the Fus3/Kss 1 pathway is not universal in all plant pathogenic fungi. Several fungi including $M$. grisea and Ustilago maydis(ubc3) did not reduce the growth rate by the disruption of the pathway (Muller et al., 1999; Xu and Hamer, 1996).

The Fus3/Kss 1 pathway is also involved in conidiation of Alternaria spp.. In A. alternata, the AaFUS3-deficient mutants could not produce fully differentiated conidia, either in culture media or in plants. The mutants only produced highly melanized hyphae with distinct septae in chains on PDA media. The addition of cAMP, yeast extracts, or glucose could not induce conidiation at all (Lin and Chung, 2010). Similar conidiation defect was also reported in Amk1-deficient mutants of A. brassicicola (Cho et al., 2007). They could not produced fully developed conidia on many media, e.g., PDA, weak PDA (1/20 concentration), and glycerol yeast extract agar (Cho et al., 2007). There are two classifications of plant pathogenic fungi related to the role of the Fus3/Kss 1 pathway on conidiation. Mutants of Fus3 with the defect in conidiation were found in many fungi such as Cryphonectria parasitica
(Choi et al., 2005), Fusarium graminearum (Jenczmionka et al., 2003), Cochliobolus heterostrophus (Lev et al., 1999) and Pyrenophora teres (Ruiz-Roldan et al., 2001), whereas those Fus 3 mutants of M. grisea (Xu and Hamer, 1996), B. cinerea (Zheng et al., 2000), Fusarium oxysporum (Di Pietro et al., 2001) and C. lagenarium (Takano et al., 2000) could produce conidia normally. These results suggest that the Fus $3 /$ Kss 1 pathway plays distinct roles for conidiation among different fungal species.

The most important and conserved function of Fus $3 /$ Kss 1 pathway can be the regulation of pathogenicity of the fungi. Most of the reported mutants of Fus3/Kss 1 homologs showed reduced or even no pathogenicity on their hosts, but the mechanisms for the lowered pathogenicity varied among different fungi (Table 1). In Alternaria spp., disruption of $A$. alternata AaFus3 or A. brassicicola Amk1 (Fus3-type MAPK) also resulted in reduced pathogenicity. The Amk1 mutants could not induce noticeable disease symptoms on Brassica oleracea even inoculations with 300 times more protoconidia than the wild type, though they were capable of infecting wounded leaves. Disruption of Amkl gene may cause the reduction even loss of the penetration ability. Further infection assay on the host leaf surface confirmed this hypothesis. Although the Amkl mutants could produce appresorium-like structure on the leaf surface, but this kind of appresorium could not penetrate leaf surface or cause tissue destruction as that of the wild type (Cho et al., 2007). The fault in appresorium production was also found in the similar mutants of $M$. grisea (Xu and Hamer, 1996), C. lagenarium (Takano et al., 2000), C. heterostrophus (Lev et al., 1999) and P. teres (Ruiz-Roldan et al., 2001), which indicated a conserved role of Fus3/Kss 1 pathway on appresorium production. Interestingly, the Amk1 mutants partially restored their pathogenicity by addition of crushed host plant leaves or nutrients like tryptone, yeast extract and bovine serum albumin, which was never reported in other fungi. These results suggest that there is another nutrientsactivated signaling pathway involving in pathogenesis. Similar to the Amk1 mutants, the AaFus3 mutants of $A$. alternata also behaved differently on intact leaves and wounded leaves havingonly pinpoint lesions on intact leaves but typical necrotic lesions on wounded leaves. The lesions on wounded leaves caused by the AaFus 3 mutants did not have significant difference in size with those caused by the wild-type strain (Lin and Chung, 2010). While in $P$. teres and C. lagenarium, Fus3/Kss 1-type MAPK mutants are nonpathogenic both on intact and wounded hosts. The difference between Alternaria and other fungi indicated the diverse roles Fus3/Kss 1 homogies played on pathogenesis in different fungi.

In Alternaria the Fus3/Kss 1 pathway also played an important role in the production of some cell-wall-degrad- 
ing enzymes (CWDE), which was only reported in F. oxysporum (Di Pietro et al., 2001), C. heterostrophus (Lev, 2003), Trichoderma atroviride (Olmedo-Monfil et al., 2002) and Trichoderma virens (Mendoza-Mendoza, 2003). Deletion the AaFus3 gene resulted in higher activities of lipase alkaline phosphatase, and cutinase, which result suggest a negative regulation of these three genes by AaFus3. But for pectinase, xylanase, and cellulase activities, there were no noticeable differences between the wild type and AaFus 3 mutants (Lin and Chung, 2010). In A. brassicicola, Amk1 also negatively regulated some CWDE such as chymotrypsin, glycosyl hydrolase, and N-acetyl glucosaminidase in the nutrient rich media (Cho et al., 2007). In the infection process, the genes of chymotrypsin, glycosyl hydrolase, and lipase showed a high expression in the wild type strain and in Amk1 mutants on wounded plants, but not on the intact ones (Cho et al., 2007). These results coincided with the pathogenicity of Amkl mutants.

\section{The High-Osmolarity Glycerol (Hogl) MAPK path- way}

The Hogl pathway is intensively studied in the budding yeast $S$. cerevisiae. By inducing the production of glycerol, the Hogl pathway thus allows yeast to adapt to high osmolarity (Brewster et al., 1993). For long time the Hogl pathway was considered to be exclusively involved in the response to extracellular osmolarity. However, subsequent studies revealed that this signal route is responsible for diverse processes of $S$. cerevisiae, such as adaptation to heat stress (Winkler et al., 2002) and citric acid stress (Lawrence et al., 2004). This pathway is also responsive to arsenite (Sotelo and Rodriguez-Gabriel, 2006) and bacterial endotoxin (Marques et al., 2006). In filamentous fungi, the Hogl-type MAPKs function in adaptation to abiotic/fungicide stress, secondary metabolism, conidiation and pathogenicity (Table 2).

The homolog of Hogl (AaHogl) in A. alternata is also involved in cellular resistance to osmotic stresses as have been reported for many other fungi. AaHogl disruption mutants were highly sensitive to potassium chloride and sodium chloride, but not to glucose, sucrose, sorbitol or mannitol. AaHogl disruption mutants also showed sensitivity to oxidative stress caused by tert-butyl-hydroxyperoxide, $\mathrm{H}_{2} \mathrm{O}_{2}$, and menadione (Lin and Chung, 2010). While in A. brassicicola, the Abhogl mutants displayed sensitivity

Table 2. Hog 1 homologs and their functions

\begin{tabular}{|c|c|c|c|c|}
\hline Fungal Species & Gene & $\begin{array}{l}\text { Genebank } \\
\text { accession no. }\end{array}$ & Functions & References \\
\hline A.alternata & AaHogl & Q52PH6 & $\begin{array}{l}\text { Adaption to osmotic and oxidative stress, fungicides susceptibility, } \\
\text { pathogenesis }\end{array}$ & (Lin and Chung, 2010) \\
\hline A.brassicicola & AbHogl & AAX86000 & $\begin{array}{l}\text { Adaption to osmotic and oxidative stress, fungicides susceptibility, } \\
\text { pathogenesis, resistance to camalexin and brassinin }\end{array}$ & (Joubert et al., 2011) \\
\hline A. fumigatus & $\operatorname{sak} A$ & XP_752664 & $\begin{array}{l}\text { Conidial germination in response to nitrogen and carbon source starva- } \\
\text { tion }\end{array}$ & (Xue et al., 2004) \\
\hline A. fumigatus & $M p k C$ & XP_753727 & Carbon source utilization & (Reyes et al., 2006) \\
\hline A. nidulans & sakA & - & stress signal transduction, sexual development and spore viability & (Kawasaki et al., 2002) \\
\hline B. bassiana & Bbhog1 & AAS77871 & $\begin{array}{l}\text { Abiotic stress resistance, pathogenicity, appressorium formation, regula- } \\
\text { tion of hydrophobicity gene expression, adhesion to insect cuticles }\end{array}$ & -(Zhang et al., 2009) \\
\hline B. cinerea & BcSakl & CAJ85638 & $\begin{array}{l}\text { Conidiation, vegetative growth, sclerotial development, host penetration, } \\
\text { oxidative stress, melanin biosynthesis }\end{array}$ & $\begin{array}{l}\text { (Liu et al., 2011, } \\
\text { Segmuller et al., 2006) }\end{array}$ \\
\hline B. oryzae & Srm1 & BAE48722 & Response to hyperosmotic stress, oxidative and UV stress & (Moriwaki et al., 2006) \\
\hline C. albicans & $\operatorname{Hog} 1$ & XP_721016 & $\begin{array}{l}\text { Oxidative stress response, chlamydospore formation, morphogenesis, } \\
\text { virulence, respiratory metabolism }\end{array}$ & $\begin{array}{l}\text { (Alonso-Monge et al., 1999, } \\
\text { 2003, 2009) }\end{array}$ \\
\hline \multicolumn{2}{|c|}{ C. heterostrophus Hogl } & BAD99295 & $\begin{array}{l}\text { Melanin biosynthesis, resistance to hyperosmotic and oxidative stress, } \\
\text { stress up-regulation of G3PPlgene, penetration }\end{array}$ & (Igbaria et al., 2008) \\
\hline C. lagenarium & Osc1 & BAD11137 & Responses to hyperosmotic stress and sensitivity to fludioxonil & (Kojima et al., 2004) \\
\hline C. parasitica & Cpmk1 & AAO27796 & $\begin{array}{l}\text { Virulence, pigmentation, conidiation, laccase production and cryparin } \\
\text { expression }\end{array}$ & (Park et al., 2004) \\
\hline F. proliferatum & Fphog1 & АBO46009 & Different abiotic stresses response, fumonisin biosynthesis & $\begin{array}{l}\text { (Ádám et al., 2008, } \\
\text { Kohut et al., 2009) }\end{array}$ \\
\hline M. grisea & Osml & AAF09475 & Osmotolerance, stress response & (Dixon et al., 1999) \\
\hline M. graminicola & Mghogl & ABD92790 & Pathogenicity, osmotolerance, fungicide resistance, yeast-like growth & (Mehrabi et al., 2006b) \\
\hline S. cerevisiae & Hog1 & NP_013214 & Osmolyte synthesis & (Gustin et al., 1998) \\
\hline $\begin{array}{l}\text { Trichoderma } \\
\text { harzianum }\end{array}$ & ThHogl & - & hyperosmotic stress response & (Delgado-Jarana et al., 2006) \\
\hline
\end{tabular}


to oxidant menadione, not to $\mathrm{H}_{2} \mathrm{O}_{2}$ (Joubert et al., 2011). In addition to osmotolerance and oxidative stress resistance, the Hogl homologs also exhibited adaption to other abiotic stresses such as heat and UV-irradiations (Ádám et al., 2008). Normally the Hogl pathway regulated accumulation of some carbohydrates in response to the hyperosmotic stresses, but the carbohydrates varies among different fungi. The accumulated carbohydrates includeglycerol in $S$. cerevisiae (Brewster et al., 1993), arabitol in M. grisea (Dixon et al., 1999), glycerol and arabitol in Candida albicans (Kayingo and Wong, 2005), erythritol and arabitol in B. bassana (Zhang et al., 2009). In Alternaria spp., there is no research on this topic and the accumulated carbohydratet is not clear.

The Hog1 MAPK pathway also plays a pivotal role in fungicides susceptibility. Hogl mutants of fungi like Fusarium proliferatum (Ádám et al., 2008), Aspergillus nidulans (Kawasaki et al., 2002), C. heterostrophus (Igbaria et al., 2008) and C. lagenarium (Kojima et al., 2004) all showed tolerant to phenylpyrrole and dicarboximide fungicides. In A. alternata, the AaHog1 mutants were also resistant to dicarboximide and phenylpyrrole fungicides, but the induced level of resistance was very small, implying another signaling pathway in response to these fungicides stress (Lin and Chung, 2010). Phenylpyrrole resistance was also found in the Abhogl mutants of A. brassicicola, and this resistance was much higher than that of AaHogl mutants (Joubert et al., 2011).

The Hog1 homologs play species-specific role in fungi pathogenesis. The Hog1-type mutants of $M$. grisea (Dixon et al., 1999), C. lagenarium (Kojima et al., 2004) and $B$. oryzae (Moriwaki et al., 2006) remained fully pathogenic on their own hosts. However, $\mathrm{MgHog} 1$ disruption mutants of M. graminicola could not infect wheat leaves (Mehrabi et al., 2006b). In C. parasitica, knockout of the Hog1 homolog cpmkl induced some hypovirulence-associated symptoms including reduction in pigmentation, conidiation, laccase production, and virulence on chestnut tree (Park et al., 2004). The Hog1 signaling pathway is also involved in Alternaria pathogenesis. Inactivation of AaHogl resulted in reduced pathogenicity of $A$. alternata on the leaves of tangerine Minneola. Both point inoculation and spray inoculation with $\mathrm{AaHog} 1$ conidial suspension produced no lesions on intact leaves, while characteristic necrotic symptom was observed on leaves inoculated with wild type strain. Loss or reduction of penetration capacity may not account for pathogenicity defect since the AaHogl mutants were also non-pathogenic on pre-wounded leaves. Inoculation with cell-free culture filtrates obtained from the AaHogl mutants or wild types showed similar results, which excluded the participation of the host-selective toxin in the reduced pathogenicity of $\mathrm{AaHog} 1$ mutants. The detoxifi- cation of reactive oxygen species or resistance to phytoalexin may be (partially) responsible for the pathogenicity defect of the AaHog1 mutants (Lin and Chung, 2010). The reduced pathogenicity was also found in Abhogl mutants of A. brassicicola. The Abhogl mutants were hypersensitive to camalexin and brassinin and had a low conidia germination rates. This defect may be partially responsible for the reduced pathogenicity (Joubert et al., 2011).

In $S$. cerevisiae, $\operatorname{Sln} 1 \mathrm{p}$, the histidine kinase (HK), works as an osmosensor and negatively regulates the HOG1 signaling pathway (Maeda et al., 1994). In filamentous fungi, the HK-mediated signal pathways were also connected with Hog1-type MAP kinase pathway, but the mode of regulation is varied in the different fungi. In $C$. heterostrophus, Dic-1, a Group III HK, positively regulates phosphorylation of the HOG1 MAPK (Yoshimi et al., 2005). While in $B$. cinerea, the histidine kinase Bos1 negatively regulated the HOG1-like MAP kinase Sak1 (Liu et al., 2008). As for A. alternata, both AaHSK1 (a two-component histidine kinase) and $A a H o g 1$ were involved in osmotic stress, but the AaHSK1-deletion mutants were hypersensitive to salt stress, but not to the high concentrations of sugar. In contrast, the $\mathrm{AaHog} 1$ mutants were resistant to salt and sensitive to sugar. The AaHSK1 mutants showed obvious resistance to oxidants and fungicides (dicarboximide and phenylpyrrole) while AaHogl mutants did not. These results suggested that the HK-mediated signal pathways work independently of the Hogl signaling pathway in regulating some processes in A. alternata. AbNik1, a twocomponent histidine kinase gene of $A$. brassicicola, upregulated the $H o g 1$ MAPK cascade which decreased fungicidal activity of ambruticin (Dongo et al., 2009). However, Joubert (2011) reported that camalexin-induced phosphorylation of AbHoglp was independent of the regulation of the AbNik1. These data suggested that the HK- and Hog1mediated signaling pathway work differently in different fungi.

\section{The Cell Wall Integrity MAPK pathway (SIt2 MAPK pathway)}

The Slt2 MAPK pathway in S. cerevisiae regulates cell wall integrity and is activated in response to external stimuli such as high temperature (Humberto et al., 1993), hypoosmolarity (Davenport et al., 1995) and cell-wall-stress agents (Ketela et al., 1999). Unlike the extensively studied Fus3/Kss1 pathways, Slt2 homologs are less well investigated in fungi. The Slt2 homologys have been shown to beinvolved in regulating cell wall integrity, pathogenicity/ virulence, conidiation, oxidative stress, melanin biosynthesis (Table 3).

As the primary function of Slt2 pathway, its homologs in 
Table 3. Slt2 homologs and their functions

\begin{tabular}{|c|c|c|c|c|}
\hline Fungal Species & Gene & $\begin{array}{l}\text { Genebank } \\
\text { accession no. }\end{array}$ & Functions & References \\
\hline A. alternata & AaSlt2 & ADC35363 & \multicolumn{2}{|c|}{$\begin{array}{l}\text { Adaption to oxidative stress,accumulation of melanin and chitin, conid- (Yago et al., 2011) } \\
\text { ial formation, pathogenicity }\end{array}$} \\
\hline A. brassicicola & AbSlt2 & AAU11317 & Adaption to oxidative stress, conidial germination, pathogenicity & (Joubert et al., 2011) \\
\hline A. fumigatus & $M p k A$ & XP_751459 & $\begin{array}{l}\text { Cell wall integrity, oxidative stress, pyomelanin formation, gliotoxin } \\
\text { production and iron adaptation }\end{array}$ & (Valiante et al., 2008) \\
\hline A. nidulans & $M p k A$ & AAD24428 & Conidial germination, polarized growth & (Bussink and Osmani, 1999) \\
\hline B. cinerea & Bmp3 & ABJ51957 & $\begin{array}{l}\text { Aprotrophic growth, conidiation, plant surface sensing, host coloniza- } \\
\text { tion, melanin biosynthesis }\end{array}$ & (Rui and Hahn, 2007) \\
\hline C. albicans & $M k c 1$ & P43068 & Cell wall construction, resistance to antifungals, virulence & $\begin{array}{l}\text { (Diez-Orejas et al., 1997, } \\
\text { Navarro-Garcia et al., 1995; } \\
\text { 1998) }\end{array}$ \\
\hline C. heterostrophus & Mps1 & ABM54149 & Melanin Biosynthesis, conidiation, virulence, female fertility & $\begin{array}{l}\text { (Eliahu et al., 2007, Igbaria } \\
\text { et al., 2008) }\end{array}$ \\
\hline C. lagenarium & Maf1 & AAL50116 & Pathogenicity, appressorium formation, conidiation & (Kojima et al., 2002) \\
\hline C. neoformans & $M p k 1$ & & Cell wall integrity, resistance to antifungals, calcineurin & (Kraus et al., 2003) \\
\hline C. purpurea & Cpmk2 & CAC87145 & Pathogenicity, conidiation, cell wall structure, virulence & (Mey et al., 2002a) \\
\hline F. graminearum & $M g v 1$ & AAM13670 & Vegetative growth, female fertility, plant infection, cell wall integrity & (Hou et al., 2002 \\
\hline M. grisea & Mps1 & AAC63682 & $\begin{array}{l}\text { Pathogenicity, cell wall integrity, pathogen penetration, appressorium } \\
\text { formation, conidiation, female fertility, }\end{array}$ & (Xu et al., 1998) \\
\hline M. graminicola & MgSlt 2 & AAY98511 & $\begin{array}{l}\text { Pathogenicity, fungicide resisitance, pycnidia formation, virulence, } \\
\text { melanisation }\end{array}$ & (Mehrabi et al., 2006a) \\
\hline S. cerevisiae & Slt2 & NP_011895 & Cell wall remodeling & (Gustin et al., 1998) \\
\hline T. virens & $\operatorname{TmkB}$ & ACD88751 & Cell wall integrity, conidiation, vegetative growth, melanin biosynthesis & (Kumar et al., 2010) \\
\hline
\end{tabular}

many pathogenic fungi also had the role of maintaining cell wall integrity in M. grisea (Xu et al., 1998), Claviceps purpurea (Mey et al., 2002a), Aspergillus fumigates (Valiante et al., 2008), A. nidulans (Bussink and Osmani, 1999), $F$. graminearum (Hou et al., 2002) and M. graminicola (Mehrabi et al., 2006a). However, in B. cinerea, the $\Delta$ bmp3 (Slt2 homologs) mutant and the wild-type showed no difference of protoplast release when treated with $\beta$-glucanase, the chitin synthase inhibitor Nikkomycin Z, and the cell-wall formation inhibitor Calcofluor-White (Rui and Hahn, 2007). Similar results were also founded in C. lagenarium (Kojima et al., 2002). These results indicate that the Slt2-related pathway is not essential for maintaining cell wall integrity in these two fungi. In A. alternata, AaSlt2 mutants were hypersensitive to cell wall-degrading enzymes and compounds. Further analysis showed that disruption of AaSlt2 resulted in less accumulation of melanin and chitin (Yago et al., 2011). Melanin locates in the fungal cell wall (Jacobson, 2000) and affects cell wall thickness, therefore, it protects fungal pathogens against stresses such as oxidants, UV radiation (Jacobson, 2000, Romero-Martinez et al., 2000), and wall-degrading enzymes (Butler et al., 2001). Did the reduced amount of melanin and chitin cause the weak cell wall of AaSlt2 mutant? This question needs further investigation. Deletion of an SLT2- homolog AbSlt2 in A. brassicicola also increased sensitivity to oxidative stress, and the AbSlt 2 mutant showed hypersensitivity to while the
AaSlt2 mutants grew normally in potato dextrose agar (PDA) containing $30 \mathrm{mM} \mathrm{H}_{2} \mathrm{O}_{2}$ (Joubert et al., 2011; Yago et al., 2011).

As have been shown in C. lagenarium (Kojima et al., 2002), C. purpurea (Mey et al., 2002a), M. grisea (Xu et al., 1998) and B. cinerea (Rui and Hahn, 2007), the Slt2 pathway was also involved in conidiation in Alternaria. In A. alternata, deletion of AaSlt 2 signiticantly reduced conidial formation, but did not affect the conidial germination. Further light microscopy examination showed that the conidia of the AaSlt2 mutants displayed an aberrant shape as less melanized, few distinct vertical and transverse septa (Yago et al., 2011). The AbSlt2 disruption mutant of $A$. brassicicola also produced deformed conidia, but unlike AaSlt2 mutants, they had normal conidiation rates and delayed germination rate (Joubert et al., 2011). Conidia of similar mutants in $M$. grisea had normal morphology and germination rates (Xu et al., 1998). These findings suggest that the Slt2 pathway takes different effects on conidiation in different fungi, although thet Slt2 pathway in several fungi is involved directly in the conidiation.

Like the Fus3/Kss 1 pathway, the Slt2 pathway is also involved in pathogenicity of Alternaria, which has been shown in other phytopathogenic fungi (Table 3 ). In $A$. alternata, spraying inoculation with conidial suspension of the wild type or the A. alternata Slt 2 mutant induced different symptoms on tangerine Minneola leaves. The lesions 
induced by the Slt 2 mutant were smaller and progressed more slowly than that induced by the wild type strain, which may be a comprehensive result of reduced production of ACT (Alternaria citri tangerine pathotype) -toxin, growth retardation and deformation of hyphal extension (Yago et al., 2011). Disruption of AbSlt2 gene also reduced the virulence of $A$. brassicicola. However, there were two different results in two independent reports as for the cause of the attenuated pathogenicity of AbSlt 2 mutants. Intact and pre-wounded leaves of $B$. oleracea were inoculated with spore suspension of wild type strain and AbSlt2 mutants. On intact leaves, these two reports showed similar result as large typical necrosis on leaves with the wild type and small lesions on leaves with the AbSlt 2 mutants. However, on pre-wounded leaves, Joubert (2011) reported that the AbSlt2 mutant still showed reduced pathogenicity compared with the wild type strain, similar results with that on intact leaves. While in the Scott's report, the AbSlt2 mutant recovered their pathogenicity on wounded leaves (Scott, 2008). There was no difference in the size of the lesions caused by the AbSlt2 mutants and the wild type strain. These results on intact leaves suggested that the reduced penetration capacity is partially responsible for the pathogenicity defect in the AbSlt2 mutants. Microscopic observations showed that conidia of the AbSlt 2 mutant only produced excessive elongation hyphae on onion epidermis and cabbage leaf surfaces and rarely formed appressoria (Joubert et al., 2011). Even the formed appressoria seemed to not be fully developed or immature (Scott, 2008). But why the pathogenicity of the AbSlt2 mutants on pre-wounded leaves behaved so differently is still questionable, which question needs to be further investigated.

An important function for AbSlt 2 can be the protection of $A$. brassicicola cells from cell wall stress caused by indolic phytoalexins. As mentioned earlier, the Abhog1-mediated signaling pathway was also involved in phytoalexins stress (Joubert et al., 2011). This feature may be a cause of the reduced virulence in AbSlt2 and Abhog 1 mutants; however, the mechanisms of the regulations by these genes are still not investigated.

\section{Concluding remarks}

There have been many researches to understand the roles of genes on the MAPK signaling pathway of Alternaria spp., especially for $A$. alternata and $A$. brassicicola. The MAPK genes, Amk1/AbSlt2/AbHog1 in A. brassicicola and AaFus3/ AaSlt2/AaHog1 in A. alternata, have been characterized and the functions have been also analyzed. Like their homologs in other plant pathogenic fungi, these MAPK pathways have some conserved roles, such as the Fus3/Kss 1-type pathway on regulation of vegetative growth, conidiation and pathogenicity, the Hogl-type pathway on adaptation to osmotic stress and fungicides susceptibility, and the Slt2type pathway on maintenance of cell wall integrity. These MAPK pathways in Alternaria, however, also have unique functions shown in the adaptation to brassicaceous indolic phytoalexins by the Slt2-type pathway.

Although some achievements have already been obtained, the research on Alternaria MAPK signaling networks is still at the beginning compared to the wealthy data of other fungi such as $S$. cerevisiae and $M$. grisea. No MAPKK and MAPKKK gene in Alternaria species has been reported, therefore, the underlying mechanism for the regulatory functions is still unknown. In the future, more studies will be required to identify the unknown MAPK cascade components, upstream activators and downstream target transcription factors, and also elucidate the regulatory networks with other signaling pathways.

\section{Acknowledgements}

This work was supported by the Young Scientist Exchange Program between the Republic of Korea and the People's Republic of China, China Postdoctoral Science Foundation, and Postdoctoral Fund of Shandong Agricultural University. Baek was supported by a grant from Systems and Synthetic Agrobiotech Center through Next-Generation BioGreen21 Program (PJ008034), RDA, Korea.

\section{References}

Adám, A. L., Kohut, G. and Hornok, L. 2008. Fphog1, a hog-type map kinase gene, is involved in multistress response in Fusarium proliferatum. J. Basic Microbiol. 48:151-159.

Alonso-Monge, R., Carvaihlo, S., Nombela, C., Rial, E. and Pla, J. 2009. The hog1 map kinase controls respiratory metabolism in the fungal pathogen Candida albicans. Microbiology 155:413-423.

Alonso-Monge, R., Navarro-Garcia, F., Molero, G., Diez-Orejas, R., Gustin, M., Pla, J., Sanchez, M. and Nombela, C. 1999. Role of the mitogen-activated protein kinase hoglp in morphogenesis and virulence of Candida albicans. J. Bacteriol. 181:3058-3068.

Alonso-Monge, R., Navarro-Garcia, F., Roman, E., Negredo, A. I., Eisman, B., Nombela, C. and Pla, J. 2003. The hog1 mitogen-activated protein kinase is essential in the oxidative stress response and chlamydospore formation in Candida albicans. Eukaryot. Cell 2:351-361.

Brachmann, A., Schirawski, J., Muller, P. and Kahmann, R. 2003. An unusual map kinase is required for efficient penetration of the plant surface by Ustilago maydis. EMBO J. 22:2199-2210.

Brewster, J. L., De Valoir, T., Dwyer, N. D., Winter, E. and Gustin, M. C. 1993. An osmosensing signal transduction pathway in yeast. Science 259:1760-1763.

Butler, M. J., Day, A. W., Henson, J. M. and Money, N. P. 2001. 
Pathogenic properties of fungal melanins. Mycologia 93:1-8.

Bussink, H. J. and Osmani, S. A. 1999. A mitogen-activated protein kinase (mpka) is involved in polarized growth in the filamentous fungus, Aspergillus nidulans. FEMS Microbiol. Lett. 173:117-125.

Chen, C., Harel, A., Gorovoits, R., Yarden, O. and Dickman, M. B. 2004. Mapk regulation of sclerotial development in Sclerotinia sclerotiorum is linked with ph and camp sensing. Mol. Plant-Microbe Interact. 17:404-413.

Chen, J., Wang, Q. and Chen, J. Y. 2000. Cek2, a novel mapk from Candida albicans complement the mating defect of fus3/ kss 1 mutant. Sheng Wu Hua Xue Yu Sheng Wu Wu Li Xue Bao (Shanghai) 32:299-304.

Chen, R. E. and Thorner, J. 2007. Function and regulation in mapk signaling pathways: Lessons learned from the yeast Saccharomyces cerevisiae. Biochim. Biophys. Acta 1773:13111340.

Cho, Y., Cramer Jr, R. A., Kim, K.-H., Davis, J., Mitchell, T. K., Figuli, P., Pryor, B. M., Lemasters, E. and Lawrence, C. B. 2007. The fus $3 / \mathrm{kss} 1$ map kinase homolog amk1 regulates the expression of genes encoding hydrolytic enzymes in Alternaria brassicicola. Fungal Genet. Biol. 44:543-553.

Choi, E. S., Chung, H. J., Kim, M. J., Park, S. M., Cha, B. J., Yang, M. S. and Kim, D. H. 2005. Characterization of the erk homologue cpmk2 from the chestnut blight fungus Cryphonectria parasitica. Microbiology 151:1349-1358.

Cousin, A., Mehrabi, R., Guilleroux, M., Dufresne, M., T, V. D. L., Waalwijk, C., Langin, T. and Kema, G. H. 2006. The map kinase-encoding gene mgfus3 of the non-appressorium phytopathogen Mycosphaerella graminicola is required for penetration and in vitro pycnidia formation. Mol. Plant Pathol. 7:269-278.

Csank, C., Schroppel, K., Leberer, E., Harcus, D., Mohamed, O., Meloche, S., Thomas, D. Y. and Whiteway, M. 1998. Roles of the Candida albicans mitogen-activated protein kinase homolog, cek1p, in hyphal development and systemic candidiasis. Infect Immun. 66:2713-2721.

Davenport, K. R., Sohaskey, M., Kamada, Y., Levin, D. E. and Gustin, M. C. 1995. A second osmosensing signal transduction pathway in yeast. Hypotonic shock activates the pkc1 protein kinase-regulated cell integrity pathway. J. Biol. Chem. 270:30157-30161.

Delgado-Jarana, J., Sousa, S., Gonzalez, F., Rey, M. and Llobell, A. 2006. Thhog1 controls the hyperosmotic stress response in Trichoderma harzianum. Microbiology 152:1687-1700.

Di Pietro, A., Garcia-Maceira, F. I., Meglecz, E. and Roncero, M. I. 2001. A map kinase of the vascular wilt fungus Fusarium oxysporum is essential for root penetration and pathogenesis. Mol. Microbiol. 39:1140-1152.

Diez-Orejas, R., Molero, G., Navarro-Garcia, F., Pla, J., Nombela, C. and Sanchez-Perez, M. 1997. Reduced virulence of Candida albicans mkcl mutants: A role for mitogen-activated protein kinase in pathogenesis. Infect Immun. 65:833-837.

Dixon, K. P., Xu, J. R., Smirnoff, N. and Talbot, N. J. 1999. Independent signaling pathways regulate cellular turgor during hyperosmotic stress and appressorium-mediated plant infec- tion by Magnaporthe grisea. Plant Cell 11:2045-2058.

Dongo, A., Bataille-Simoneau, N., Campion, C., Guillemette, T., Hamon, B., Iacomi-Vasilescu, B., Katz, L. and Simoneau, P. 2009. The group III two-component histidine kinase of filamentous fungi is involved in the fungicidal activity of the bacterial polyketide ambruticin. Appl Environ Microbiol 75:127134.

Dufresne, M. and Osbourn, A. E. 2001. Definition of tissue-specific and general requirements for plant infection in a phytopathogenic fungus. Mol. Plant-Microbe Interact. 14:300-307.

Eisman, B., Alonso-Monge, R., Roman, E., Arana, D., Nombela, C. and Pla, J. 2006. The cek1 and hog1 mitogen-activated protein kinases play complementary roles in cell wall biogenesis and chlamydospore formation in the fungal pathogen Candida albicans. Eukaryot. Cell 5:347-358.

Eliahu, N., Igbaria, A., Rose, M. S., Horwitz, B. A. and Lev, S. 2007. Melanin biosynthesis in the maize pathogen Cochliobolus heterostrophus depends on two mitogen-activated protein kinases, chk1 and mps1, and the transcription factor $\mathrm{cmrl}$. Eukaryot. Cell 6:421-429.

Guo, J., Dai, X., Xu, J. R., Wang, Y., Bai, P., Liu, F., Duan, Y., Zhang, H., Huang, L. and Kang, Z. 2011. Molecular characterization of a fus3/kss1 type mapk from Puccinia striiformis $\mathrm{f}$. Sp. Tritici, psmapk1. PLoS One 6:e21895.

Gustin, M. C., Albertyn, J., Alexander, M. and Davenport, K. 1998. Map kinase pathways in the yeast Saccharomyces cerevisiae. Microbiol. Mol. Biol. Rev. 62:1264-1300.

Hou, Z., Xue, C., Peng, Y., Katan, T., Kistler, H. C. and Xu, J. R. 2002. A mitogen-activated protein kinase gene (mgvl) in Fusarium graminearum is required for female fertility, heterokaryon formation, and plant infection. Mol. Plant-Microbe. Interact. 15:1119-1127.

Humberto, M., Javier, A., Miguel, S., María, M. and César, N. 1993. Activity of the yeast MAP kinase homologue Sit2 is critically required for cell integrity at $37^{\circ} \mathrm{C}$. Mol. Gen. Genet. 241:177-184.

Igbaria, A., Lev, S., Rose, M. S., Lee, B. N., Hadar, R., Degani, O. and Horwitz, B. A. 2008. Distinct and combined roles of the map kinases of Cochliobolus heterostrophus in virulence and stress responses. Mol. Plant-Microbe. Interact. 21:769-780.

Jacobson, E. S. 2000. Pathogenic roles for fungal melanins. Clin. Microbiol. Rev. 13:708-717.

Jenczmionka, N. J., Maier, F. J., Losch, A. P. and Schafer, W. 2003. Mating, conidiation and pathogenicity of Fusarium graminearum, the main causal agent of the head-blight disease of wheat, are regulated by the map kinase gpmk1. Curr. Genet. 43:87-95.

Joubert, A., Bataille-Simoneau, N., Campion, C., Guillemette, T., Hudhomme, P., Iacomi-Vasilescu, B., Leroy, T., Pochon, S., Poupard, P. and Simoneau, P. 2011a. Cell wall integrity and high osmolarity glycerol pathways are required for adaptation of Alternaria brassicicola to cell wall stress caused by brassicaceous indolic phytoalexins. Cell. Microbiol. 13:62-80.

Jun, S.-C., Lee, S.-J., Park, H.-J., Kang, J.-Y., Leem, Y.-E., Yang, T.-H., Chang, M.-H., Kim, J.-M., Jang, S.-H., Kim, H.-G., Han, D.-M., Chae, K.-S. and Jahng, K.-Y. 2011. The mpkb 
map kinase plays a role in post-karyogamy processes as well as in hyphal anastomosis during sexual development in Aspergillus nidulans. J. Micro. 49:418-430.

Kawasaki, L., Sanchez, O., Shiozaki, K. and Aguirre, J. 2002. Saka map kinase is involved in stress signal transduction, sexual development and spore viability in Aspergillus nidulans. Mol. Microbiol. 45:1153-1163.

Kayingo, G. and Wong, B. 2005. The map kinase hog1p differentially regulates stress-induced production and accumulation of glycerol and d-arabitol in Candida albicans. Microbiology 151:2987-2999.

Ketela, T., Green, R. and Bussey, H. 1999. Saccharomyces cerevisiae $\mathrm{Mid} 2 \mathrm{p}$ is a potential cell wall stress sensor and upstream activator of the PKC1-MPK1 cell integrity pathway. J. Bacteriol. 181:3330-3340.

Kiel, C., Yus, E. and Serrano, L. 2010. Engineering signal transduction pathways. Cell 140:33-47.

Kohut, G., dám, A. L., Fazekas, B. and Hornok, L. 2009. N-starvation stress induced fum gene expression and fumonisin production is mediated via the hog-type mapk pathway in Fusarium proliferatum. Int. J. Food Microbiol. 130:65-69.

Kojima, K., Kikuchi, T., Takano, Y., Oshiro, E. and Okuno, T. 2002. The mitogen-activated protein kinase gene mafl is essential for the early differentiation phase of appressorium formation in Colletotrichum lagenarium. Mol. Plant-Microbe. Interact. 15:1268-1276.

Kojima, K., Takano, Y., Yoshimi, A., Tanaka, C., Kikuchi, T. and Okuno, T. 2004. Fungicide activity through activation of a fungal signalling pathway. Mol. Microbiol. 53:1785-1796.

Kraus, P. R., Fox, D. S., Cox, G. M. and Heitman, J. 2003. The Cryptococcus neoformans map kinase mpk1 regulates cell integrity in response to antifungal drugs and loss of calcineurin function. Mol. Microbiol. 48:1377-1387.

Kumar, A., Scher, K., Mukherjee, M., Pardovitz-Kedmi, E., Sible, G. V., Singh, U. S., Kale, S. P., Mukherjee, P. K. and Horwitz, B. A. 2010. Overlapping and distinct functions of two Trichoderma virens map kinases in cell-wall integrity, antagonistic properties and repression of conidiation. Biochem. Biophys. Res. Commun. 398:765-770.

Lawrence, C. L., Botting, C. H., Antrobus, R. and Coote, P. J. 2004. Evidence of a new role for the high-osmolarity glycerol mitogen-activated protein kinase pathway in yeast: Regulating adaptation to citric acid stress. Mol. Cell. Biol. 24:3307-3323.

Lengeler, K. B., Davidson, R. C., D'souza, C., Harashima, T., Shen, W. C., Wang, P., Pan, X., Waugh, M. and Heitman, J. 2000. Signal transduction cascades regulating fungal development and virulence. Microbiol. Mol. Biol. Rev. 64:746-785.

Lev, S. and Horwitz, B. A. 2003. A mitogen-activated protein kinase pathway modulates the expression of two cellulase genes in Cochliobolus heterostrophus during plant infection. Plant Cell 15:835-844.

Lev, S., Sharon, A., Hadar, R., Ma, H. and Horwitz, B. A. 1999. A mitogen-activated protein kinase of the corn leaf pathogen Cochliobolus heterostrophus is involved in conidiation, appressorium formation, and pathogenicity: Diverse roles for mitogen-activated protein kinase homologs in foliar pathogens.
Proc. Natl. Acad Sci. USA 96:13542-13547.

Li, D., Piotr Bobrowicz, Heather H. Wilkinson and Daniel J. Ebbole 2005. A mitogen-activated protein kinase pathway essential for mating and contributing to vegetative growth in Neurospora crassa. Genetics 170:1091-1104.

Lin, C. H. and Chung, K. R. 2010. Specialized and shared functions of the histidine kinase- and hog1 map kinase-mediated signaling pathways in Alternaria alternata, a filamentous fungal pathogen of citrus. Fungal Genet. Biol. 47:818-827.

Liu, W., Leroux, P. and Fillinger, S. 2008. The hog1-like map kinase sak 1 of Botrytis cinerea is negatively regulated by the upstream histidine kinase bos1 and is not involved in dicarboximide- and phenylpyrrole-resistance. Fungal Genet. Biol. 45:1062-1074.

Liu, W., Soulie, M. C., Perrino, C. and Fillinger, S. 2011. The osmosensing signal transduction pathway from Botrytis cinerea regulates cell wall integrity and map kinase pathways control melanin biosynthesis with influence of light. Fungal Genet. Biol. 48:377-387.

Maeda, T., Wurgler-Murphy, S. M. and Saito, H. 1994. A twocomponent system that regulates an osmosensing MAP kinase cascade in yeast. Nature 369:242-245.

Marques, J. M., Rodrigues, R. J., De Magalhaes-Sant'ana, A. C. and Goncalves, T. 2006. Saccharomyces cerevisiae hog1 protein phosphorylation upon exposure to bacterial endotoxin. $J$. Biol. Chem. 281:24687-24694.

May, G. S., Xue, T., Kontoyiannis, D. P. and Gustin, M. C. 2005. Mitogen activated protein kinases of Aspergillus fumigatus. Med. Mycol. 43 Suppl 1:S83-86.

Mehrabi, R., Van Der Lee, T., Waalwijk, C. and Gert, H. J. $2006 a$. Mgsit2, a cellular integrity map kinase gene of the fungal wheat pathogen Mycosphaerella graminicola, is dispensable for penetration but essential for invasive growth. Mol. PlantMicrobe Interact. 19:389-398.

Mehrabi, R., Zwiers, L. H., De Waard, M. A. and Kema, G. H. 2006b. Mghog1 regulates dimorphism and pathogenicity in the fungal wheat pathogen Mycosphaerella graminicola. Mol Plant-Microbe Interact 19:1262-1269.

Mendoza-Mendoza, A. 2003. Enhanced biocontrol activity of trichoderma through inactivation of a mitogen-activated protein kinase. Proc. Nat. Acad. Sci. 100:15965-15970.

Mey, G., Held, K., Scheffer, J., Tenberge, K. B. and Tudzynski, P. 2002a. Cpmk2, an slt2-homologous mitogen-activated protein (map) kinase, is essential for pathogenesis of Claviceps purpurea on rye: Evidence for a second conserved pathogenesisrelated map kinase cascade in phytopathogenic fungi. Mol. Microbiol. 46:305-318.

Mey, G., Oeser, B., Lebrun, M. H. and Tudzynski, P. 2002b. The biotrophic, non-appressorium-forming grass pathogen Claviceps purpurea needs a fus3/pmk1 homologous mitogen-activated protein kinase for colonization of rye ovarian tissue. Mol. Plant-Microbe Interact. 15:303-312.

Moriwaki, A., Kihara, J., Mori, C. and Arase, S. 2007. A map kinase gene, bmk1, is required for conidiation and pathogenicity in the rice leaf spot pathogen Bipolaris oryzae. Microbiol. Res. 162:108-114. 
Moriwaki, A., Kubo, E., Arase, S. and Kihara, J. 2006. Disruption ofsrm 1, a mitogen-activated protein kinase gene, affects sensitivity to osmotic and ultraviolet stressors in the phytopathogenic fungus Bipolaris oryzae. FEMS Microbiol. Lett. 257: 253-261.

Mukherjee, P. K., Latha, J., Hadar, R. and Horwitz, B. A. 2003. TmkA, a mitogen-activated protein kinase of Trichoderma virens, is involved in biocontrol properties and repression of conidiation in the dark. Eukaryot. Cell 2:446-455.

Muller, P., Aichinger, C., Feldbrugge, M. and Kahmann, R. 1999. The map kinase kpp2 regulates mating and pathogenic development in Ustilago maydis. Mol. Microbiol. 34:1007-1017.

Navarro-Garcia, F., Alonso-Monge, R., Rico, H., Pla, J., Sentandreu, R. and Nombela, C. 1998. A role for the map kinase gene mkc1 in cell wall construction and morphological transitions in Candida albicans. Microbiology 144 ( Pt 2):411-424.

Navarro-Garcia, F., Sanchez, M., Pla, J. and Nombela, C. 1995. Functional characterization of the $\mathrm{mkcl}$ gene of Candida albicans, which encodes a mitogen-activated protein kinase homolog related to cell integrity. Mol. Cell. Biol. 15:21972206.

Olmedo-Monfil, V., Mendoza-Mendoza, A., Gomez, I., Cortes, C. and Herrera-Estrella, A. 2002. Multiple environmental signals determine the transcriptional activation of the mycoparasitism related gene prb1 in Trichoderma atroviride. Mol. Genet. Genomics 267:703-712.

Park, S. M., Choi, E. S., Kim, M. J., Cha, B. J., Yang, M. S. and Kim, D. H. 2004. Characterization of hog1 homologue, cpmk1, from Fryphonectria parasitica and evidence for hypovirus-mediated perturbation of its phosphorylation in response to hypertonic stress. Mol. Microbiol. 51:1267-1277.

Rauyaree, P., Ospina-Giraldo, M. D., Kang, S., Bhat, R. G., Subbarao, K. V., Grant, S. J. and Dobinson, K. F. 2005. Mutations in vmk1, a mitogen-activated protein kinase gene, affect microsclerotia formation and pathogenicity in Verticillium dahliae. Curr. Genet. 48:109-116.

Reyes, G., Romans, A., Nguyen, C. K. and May, G. S. 2006. Novel mitogen-activated protein kinase mpkc of Aspergillus fumigatus is required for utilization of polyalcohol sugars. Eukaryot. Cell 5:1934-1940.

Romero-Martinez, R., Wheeler, M., Guerrero-Plata, A., Rico, G. and Torres-Guerrero, H. 2000. Biosynthesis and functions of melanin in Sporothrix schenckii. Infect. Immun. 68:36963703.

Rui, O. and Hahn, M. 2007. The slt2-type map kinase bmp3 of Botrytis cinerea is required for normal saprotrophic growth, conidiation, plant surface sensing and host tissue colonization. Mol. Plant Pathol. 8:173-184.

Ruiz-Roldan, M. C., Maier, F. J. and Schafer, W. 2001. Ptk1, a mitogen-activated-protein kinase gene, is required for conidiation, appressorium formation, and pathogenicity of Pyrenophora teres on barley. Mol. Plant-Microbe Interact. 14:116125.

Scott, D. C. 2009. The cell wall integrity-associated MAP kinase homolog, AbSlt2 in the necrotrophic fungus Alternaria brassicicola is required for pathogenicity of Brassicas. Masters
Thesis, Virginia Polytechnic Institute and State University. Available at: http://scholar.lib.vt.edu/thesis/available/etd02102009-234303.

Segmuller, N., Ellendorf, U., Tudzynski, B. and Tudzynski, P. 2006. Bcsak1, a stress-activated mitogen-activated protein kinase, is involved in vegetative differentiation and pathogenicity in Botrytis cinerea. Eukaryot. Cell 6:211-221.

Sobiya, S., Ruqeyah, A. M. and Shazia, S. 2012. Cymbopogon citrates: A remedy to control selected Alternaria species $J$. Med. Plants Res. 6:1879-1885.

Solomon, P. S., Waters, O. D. C., Simmonds, J., Cooper, R. M. and Oliver, R. P. 2005. The mak2 map kinase signal transduction pathway is required for pathogenicity in Stagonospora nodorum. Curr. Genet. 48:60-68.

Sotelo, J. and Rodriguez-Gabriel, M. A. 2006. Mitogen-activated protein kinase hog1 is essential for the response to arsenite in Saccharomyces cerevisiae. Eukaryot. Cell 5:1826-1830.

Takano, Y., Kikuchi, T., Kubo, Y., Hamer, J. E., Mise, K. and Furusawa, I. 2000. The Colletotrichum lagenarium map kinase gene cmk1 regulates diverse aspects of fungal pathogenesis. Mol. Plant-Microbe Interact. 13:374-383.

Valiante, V., Heinekamp, T., Jain, R., Härtl, A. and Brakhage, A. A. 2008. The mitogen-activated protein kinase mpka of Aspergillus fumigatus regulates cell wall signaling and oxidative stress response. Fungal Genet. Biol. 45:618-627.

Verma, P. R. and Saharan, G. S. 1994. Monograph on Alternaria diseases of crucifers. Agriculture Canada Research Station, Saskatoon, Technical Bulletin No.1994-6E: 162.

Winkler, A., Arkind, C., Mattison, C. P., Burkholder, A., Knoche, K. and Ota, I. 2002. Heat stress activates the yeast high-osmolarity glycerol mitogen-activated protein kinase pathway, and protein tyrosine phosphatases are essential under heat stress. Eukaryot. Cell 1:163-173.

Xu, J. R. and Hamer, J. E. 1996. Map kinase and camp signaling regulate infection structure formation and pathogenic growth in the rice blast fungus Magnaporthe grisea. Genes Dev. 10:2696-2706.

Xu, J. R., Staiger, C. J. and Hamer, J. E. 1998. Inactivation of the mitogen-activated protein kinase mps 1 from the rice blast fungus prevents penetration of host cells but allows activation of plant defense responses. Proc. Nat.l Acad. Sci. USA 95:1271312718.

Xue, T., Nguyen, C. K., Romans, A. and May, G. S. 2004. A mitogen-activated protein kinase that senses nitrogen regulates conidial germination and growth in Aspergillus fumigatus. Eukaryot. Cell 3:557-560.

Yago, J. I., Lin, C. H. and Chung, K. R. 2011. The slt2 mitogenactivated protein kinase-mediated signalling pathway governs conidiation, morphogenesis, fungal virulence and production of toxin and melanin in the tangerine pathotype of Alternaria alternata. Mol. Plant Pathol. 12:653-665.

Yoshimi, A., Kojima, K., Takano, Y. and Tanaka, C. 2005. Group III histidine kinase is a positive regulator of hog1-type mitogen-activated protein kinase in filamentous fungi. Eukaryot. Cell 4:1820-1828.

Zhang, Y., Zhang, J., Jiang, X., Wang, G., Luo, Z., Fan, Y., Wu, Z. 
and Pei, Y. 2010. Requirement of a mitogen-activated protein kinase for appressorium formation and penetration of insect cuticle by the entomopathogenic fungus Beauveria bassiana. Appl. Environ. Microb. 76:2262-2270.

Zhang, Y., Zhao, J., Fang, W., Zhang, J., Luo, Z., Zhang, M., Fan, Y. and Pei, Y. 2009. Mitogen-activated protein kinase hog1 in the entomopathogenic fungus Beauveria bassiana regulates environmental stress responses and virulence to insects. Appl. Environ. Microb. 75:3787-3795.

Zheng, L., Campbell, M., Murphy, J., Lam, S. and Xu, J.-R. 2000. Thebmplgene is essential for pathogenicity in the gray mold fungus Botrytis cinerea. Mol. Plant-Microbe Interact. 13:724732. 